\title{
SDI INDUSTRY: AN EXTEND-BASED TOOL FOR CONTINUOUS AND HIGH-SPEED MANUFACTURING
}

\author{
Andrew J. Siprelle \\ Richard A. Phelps \\ M. Michelle Barnes \\ Simulation Dynamics, Inc. \\ 416 High Street \\ Maryville, Tennessee 37804-5836, U.S.A.
}

\begin{abstract}
Continuous or high-speed manufacturing equipment is an integral part of the food and beverage, pharmaceutical, and consumer products industries. For many years, these industries have tried to use simulation to improve their processes with less than desired results. In the past, aggregation or continuous modeling techniques have been used which resulted in largely inaccurate and slow models.

SDI Industry, the first simulation tool designed specifically for this type of process, is built from the highly popular Extend simulation package. It provides a unique architecture for simulating these industries and the complex control logic associated with them. Organizations use SDI Industry to evaluate scheduling strategies and plant configurations, find bottlenecks, and improve throughput. Industry provides an integrated database which allows the simulation fast access to data for failures, changeovers, and many other aspects of operation.
\end{abstract}

\section{INTRODUCTION}

SDI Industry is used for throughput analysis, debottlenecking, and capacity analysis for manufacturing operations, lines and whole plants. SDI Industry supports throughput analysis from viewpoints of both engineering and business management. Engineers can use SDI Industry to evaluate equipment tradeoffs involving, for example, speed, changeover time, reliability, utilization, buffer sizing, product routing, and even sequence or distribution control logic. Business managers can use Industry to evaluate product mix, crewing and over-time, warehouse space, schedules, and even scheduling strategies. An embedded simulation database allows easy management of material and product characteristics, equipment data, schedules, and reports.

SDI Industry has been applied successfully to model whole plants in the industries of consumer products, foods, and pharmaceuticals. SDI Industry is unique in its emphasis on applying throughput analysis to batch, continuous and high-speed operations typical of the industries mentioned above. These industries are typically comprised of "making" or "processing" operations followed by high-speed packaging operations. They represent the standard old making-packing facility. Making produces bulk materials, pastes, or liquids by mixing and processing ingredients in steps that are characterized as continuous, semi-continuous, or batch. The physical gear in such operations typically consists of tanks, conveyors, mixers, dryers, extruders, and pipes. Downstream from the making operation is packaging, where the material is filled at high speed into containers, then sealed, labeled, palletized, etc. Making operations produce different flavors of base product while packaging operations further differentiate the product by packing it into different containers with different labels.

\section{EVOLUTION OF SDI INDUSTRY}

Before SDI Industry, throughput analysis practitioners in these industries have had only two kinds of choices in simulation tools. Neither kind has been adequate. Throughput analysis simulation for these industries requires a shift in focus to the modeling of material that is aptly referred to as "stuff" and not pieces or parts. The inadequacy in tools arises from the issue of simulating the flow of this "stuff" at an appropriate level of detail.

Physical process simulation (also loosely referred to as "continuous") has been routinely applied by chemical engineers. In these industries, and most particularly in chemicals industries, physical process simulation is used routinely for unit operations analysis, where the emphasis is much more engineering-focused. These simulations are used to study issues like energy balance, mass flow circularity (e.g., in distillation columns), and material constituent reactions or property feedback (e.g., temperature or pressure). 
For throughput analysis, the continuous kind of simulation is more than up to the task of modeling the continuous flow of material. In continuous systems, material flow is simulated as rates, and mass balance is a fundamental requirement. Obviously, basic mass balance is also a requirement for throughput analysis. Unfortunately this kind of simulation is stuck at a level of depth that is too detailed. In many cases in these industries, this level of detail is inappropriate to the industry as a whole (e.g., some basic foods, beverages, or consumer products), or inappropriate to the concerns, or the phase and scope of analysis. For example, these systems often require the model builder to fill in detailed material property and equipment settings to enable the simulation to calculate energy balances and material flow dynamics or chemical reactions. Even in cases where a plant employs a distillation column, a practitioner of throughput analysis would most likely want to take the simple view of such a piece of equipment as just a mass flow splitter.

Discrete event simulation has been routinely applied by industrial engineers and business managers. Discrete event simulation is the classical tool of choice for studying throughput, where broader questions of product mix, scheduling, and reliability come into play. Also, discrete event simulation is used to study plant operations over a broader scope than continuous simulations; e.g., a whole plant versus a single unit operation, and longer run periods; e.g., several months or many runs of different products versus a start-up/equilibrating kind of situation. Unfortunately, classical discrete event simulation emphasizes pieces and parts plants and not continuous ones. There are work-arounds (Sturrock and Drake, 1996) that slow the simulation down or that make critical tradeoffs between accuracy and model run speed. While these practical considerations are most important, the unnaturalness of the work-arounds provide enough headaches as to yield the practice unworkable.

In addition, discrete event simulation has never spoken well to the needs of industries whose product flow is so fast that, when viewing it from a throughput analysis perspective it might as well be continuous. For example, high speed bottling or canning operations, while pieces/parts operations, can be readily viewed as a class of continuous flow problem. The task of building efficient models for these industries requires the same work-arounds discussed above (Siprelle and Phelps, 1997).

\section{OVERVIEW OF SDI INDUSTRY}

SDI Industry is a graphical, interactive, industrial simulation program built using the popular Extend simulation package, which is developed by Imagine That. SDI Industry has evolved from Simulation Dynamics' extensive model building experience in the food and beverage, consumer products, and pharmaceutical industries (Siprelle, 1995).

SDI Industry provides Extend with a structure that enables rapid modeling of industrial processes. It provides a hierarchical framework of model templates; flow blocks to model material flows; control blocks to manage the flow; and an integrated database in which to store management information.

SDI Industry introduces a structure to general model building that exploits the hierarchical nature of Extend. This structure makes it easier to rapidly develop reusable models and model components. The task of model building with SDI Industry is divided into four defined hierarchical levels: Equipment (or process); Operations; Systems; and Plant. Each model level has its own template that guides construction, and process management blocks which control the process at that level and interface with the levels above and below.

SDI Industry's flow blocks model any kind of material flow, from parts and pieces to bulk flow and high-speed production. Flow blocks convey rate information between blocks, account for mass, and facilitate blocking and starving mechanisms through an advanced messagepassing system. This message- passing system is fully compatible with the existing Extend message architecture (Lamperti and Krahl, 1997). In addition, flow can be converted back and forth between items and bulk flow. For example, a tote container of toothpaste can be treated as an item when you are manipulating the tote, and then converted to a bulk material to flow through packaging processes. Tubes of toothpaste can be aggregated at a palletizer, and pallets can be sent forward as items.

Levels within SDI Industry are designed so that standardized controllers handle issues specific to each level. Equipment level controllers deal with the dynamics of processes such as failure, repair, and batch cycle control. Operation Level controllers manage changeovers and set process characteristics such as bin sizes or equipment rates which may be based on material characteristics. System level controllers specify and read production/shift schedules, and coordinate operation startup and shutdown sequence as product changes occur. Plant level controllers manage distribution of product between systems, and coordinate their schedules. 


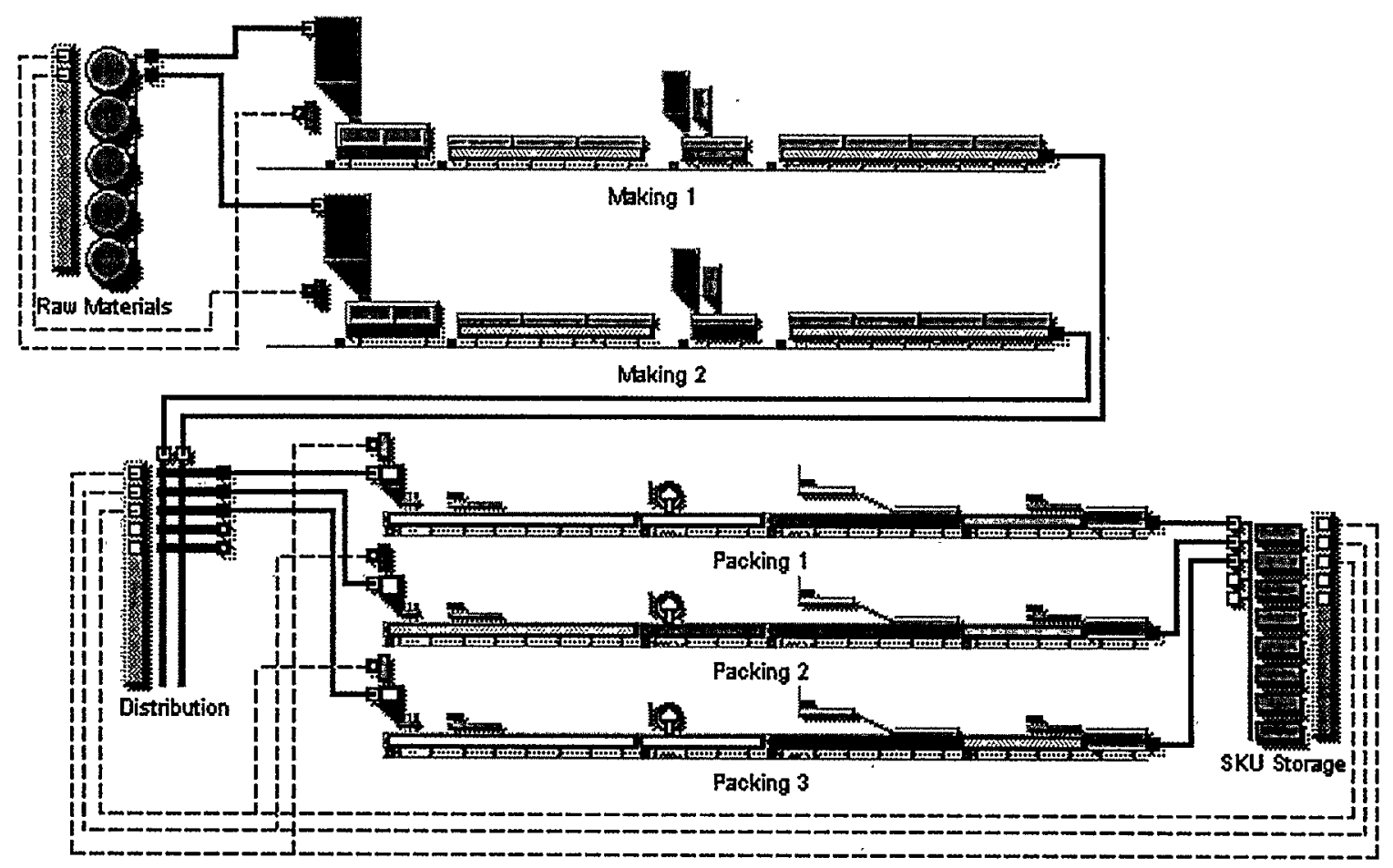

Figure 1: View of Complete Plant

SDI Industry incorporates an integrated relational database that is used to store and manage data centrally. A central database eliminates the need to search for critical data buried in the model. Standardized tables within the database support the data requirements of the control blocks at each level. Materials and Brands Tables provide information about the products to be run; an Equipment Failure Table provides Time Between Failure and Time To Repair data; Changeover Tables provide matrices of changeover times; Shift Schedule Tables provide alternative crewing schedules; and Production Schedule Tables provide specific run sequences. Report Tables can be customized to gather information as the model runs.

\section{HIERARCHICAL LEVELS OF SDI INDUSTRY}

SDI Industry is very much about the levels of plant, system operation, and equipment. Built into the levels is a philosophy or vision of how manufacturing plants work. Each level has a clear definition, rules for what happens there, and set of blocks that are usually found there. In addition, SDI Industry supplies pre-built templates for the different levels, which make building models easier. In fact, if you are building a model, you can start with a plant template, and then modify the underlying levels to represent your facility.

\subsection{Plant Level}

Figure 1 shows a model of a plant comprised of a Raw Material system, 2 Making (processing) systems, a Distribution system, 3 Packing systems, and finished SKU Storage. Each of these systems is contained in a hierarchical block. Since these blocks are hierarchical, we can double-click on a system, like the block labeled "Making 1," to expose the details of that system. The system hierarchical blocks can be easily duplicated to add new Making or Packing lines.

A Detailed System Report from the database is shown in Figure 2 on the following page. The model database can be opened to view tables or configure custom reports from the Extend menu. Tabs within the database are used to group similar kinds of tables. Drop-down menus facilitate selection of different systems and materials within the tables.

\subsection{System Level}

Double-clicking on the Making 1 system hierarchical block reveals the underlying detail shown in Figure 3 on the following page. Making 1 consists of three operations; a Mixer, an Agglomerator, and a Pre-dryer. The System Control block manages the flow of product through these operations based upon a production and shift schedule selected within the controller. The total amount of product 
Siprelle, Phelps and Barnes

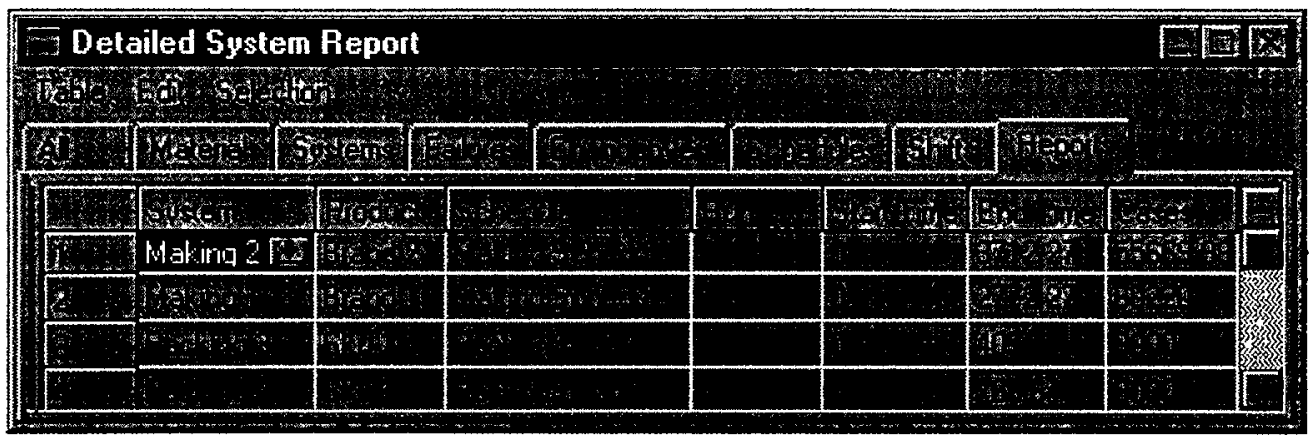

Figure 2: Detailed System Report

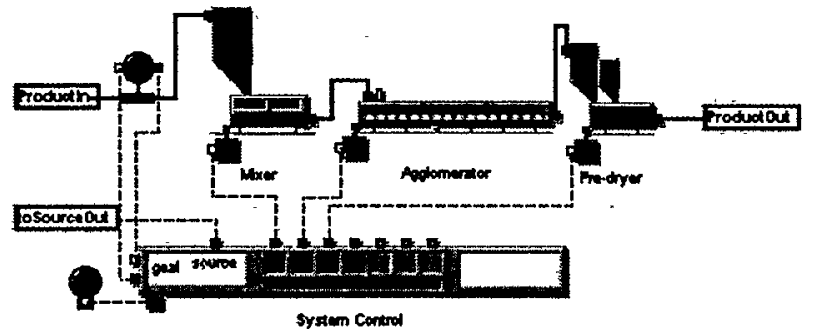

Figure 3: System Level Detail View

produced by the system is monitored, and changeovers triggered as production goals are met.

Schedules can be built in a standard format with the assistance of a wizard or populated with data directly from an Excel spreadsheet. SDI Industry supports the concept of dependent schedules, so that runs within the Packing schedule can be fed by runs within an upstream Making schedule. The database view in Figure 4 shows a typical Making schedule.

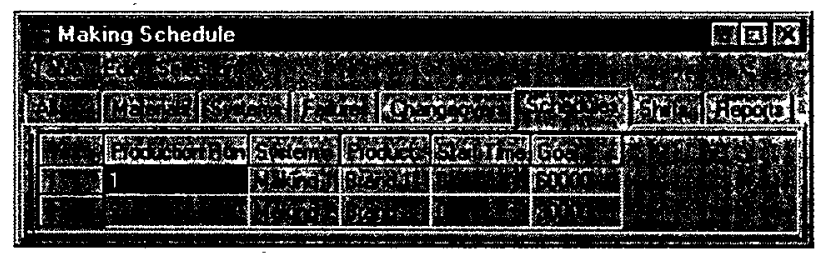

Figure 4: Typical Making Schedule

\subsection{Operation Level}

Each of the Operations is a hierarchical block. Double clicking on the Agglomerator block reveals the details of the Agglomerator operation, shown in Figure 5. The Operation Controller block at this level passes the prior and current product data and shift status information down to the Equipment level, and passes operation status information back up to the System Level. The Changeovers controller determines the changeover time associated with product changeovers as they occur. Figure 6 shows the changeover matrix referred to by this controller. The Rate

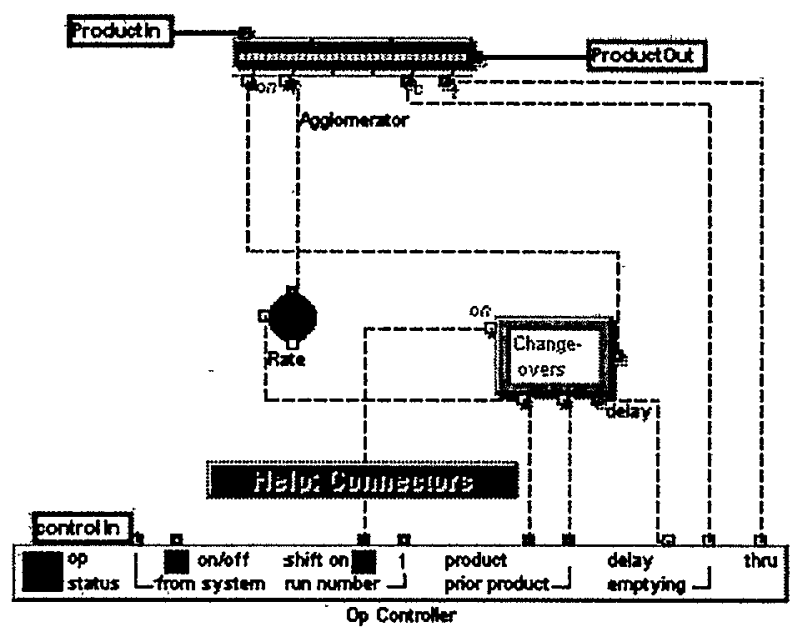

Figure 5: Operational Detail View

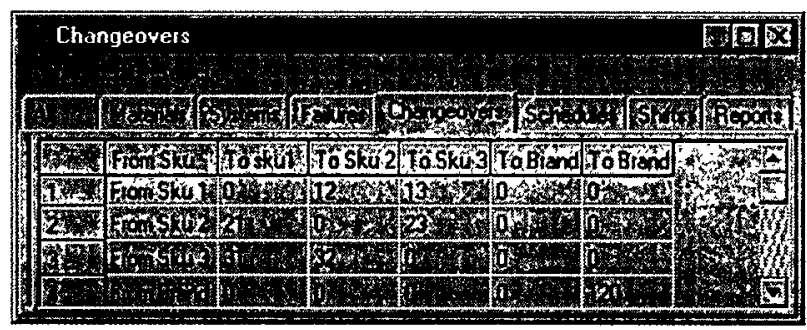

Figure 6: Equipment Changeover Detail View

controller uses the product SKU number to lookup the appropriate production rate for this Operation.

\subsection{Equipment Level}

Double-clicking on the Agglomerator block reveals the Equipment detail shown in Figure 7 on the following page. This detail shows the flow blocks which model the actual operation of the Agglomerator. The Agglomerator is a constrained process with a defined material capacity, which can be modeled with just two flow blocks. The Process controller manages the Constraint block based on 


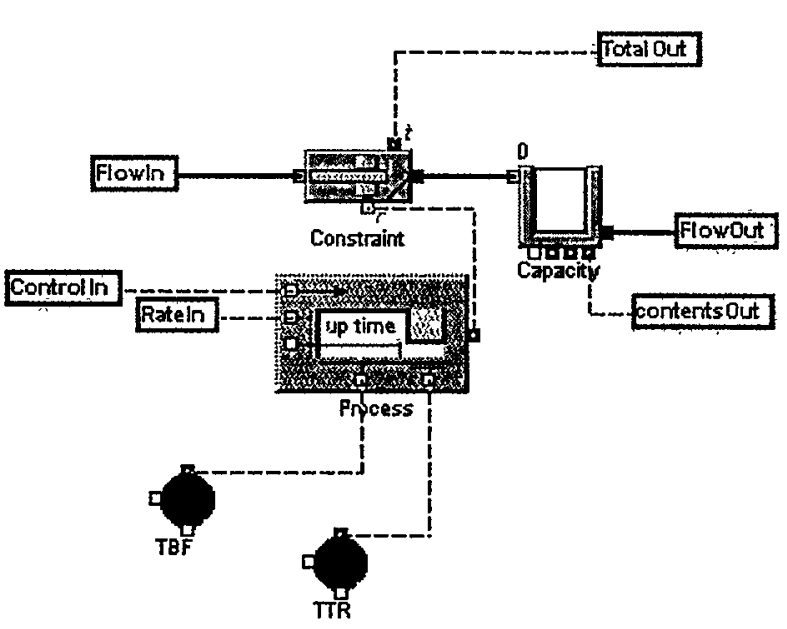

Figure 7: Equipment Level Detail View

signals it receives from the TBF and TTR controllers and the operation controller. These signals are converted into rate information for the constraint block. Figure 8 shows the database input screen for inputting the failure data for the equipment in the model.

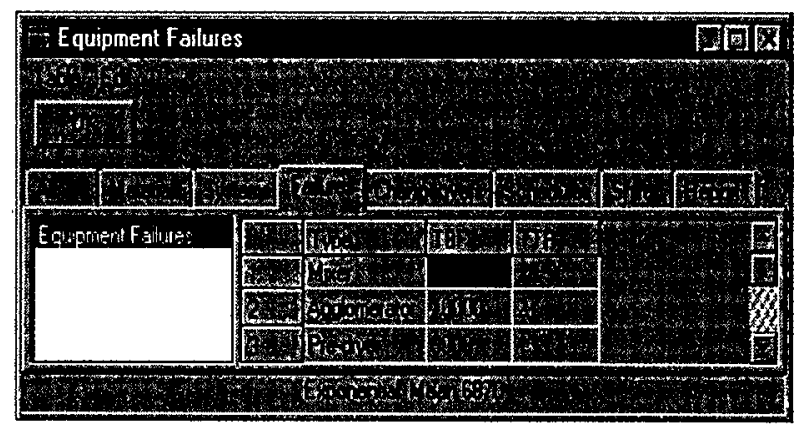

Figure 8: Equipment Failure Detail View

\section{DISTRIBUTION AND SCHEDULING WITHIN SDI INDUSTRY}

As a user works through the different hierarchical levels of SDI Industry in the process of building a model, one will eventually get to the plant level. At this topmost level, decisions must be made about two very important issues which have not been discussed much so far: distribution and scheduling. Distribution usually refers to the piping between the making and packing sections of a plant. Scheduling refers to how the material flowing through the model is controlled through time.

\subsection{Distribution}

As mentioned above, distribution is most often the manner in which material moves from the making (front-end) part of a plant to the packing (back-end) part of a plant. Distribution functions can also be required before the making portion of a plant (multiple incoming streams of raw material) or at the end of the packing part (multiple outgoing streams for shipment - trucks, railroad cars, barges, wheelbarrows). A generic plant diagram shown below illustrates two source (making) systems feeding two user (packing) systems. In SDI Industry there are three basic distribution models from which an endless variety of plant configurations can be built.

Figure 9: Generic Plant Diagram

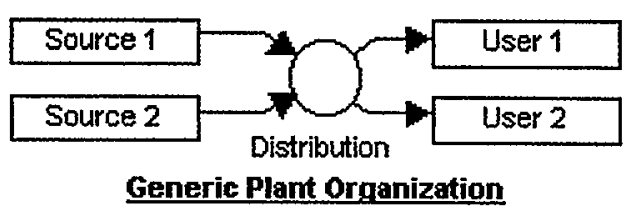

The first distribution model is continuous. In continuous distribution, the product of one source system feeds one or more user system on a continuous basis. There can be only one connection between each system and the distribution system. A given user system may draw several production runs in sequence from one source run, but when a source run ends, all of the user runs that it is feeding must end. The relationships among these runs must be defined in the production schedules for source and user systems. The distribution system itself is responsible for balancing flow among the user systems being fed by each source, and shutting down flow from each source when the user systems it is feeding are overloaded.

The second distribution model is material. In material distribution, source systems feed material into designated storage bins. There is one storage bin for each material that flows through the materials distributor. Each user line requests one or more specific material. There can be more than one connection from the distribution system to a user system.

The third distribution model is batch. In batch distribution, source systems produce batches of material which are stored in tact. These batches are then called for by number by the user systems when the schedule dictates.

SDI Industry contains pre-built blocks which model each of these types of distribution systems. The blocks can be combined to model plants which are hybrid representations of the above distribution systems. 


\subsection{Scheduling}

Production schedules in SDI Industry are defined at the system level of a model. Industry provides a standard way of defining and storing schedules, and coordinates the running of the schedules. A production schedule determines the sequence of production operations. The schedule specifies the product being produced, when it is produced and either the quantity or the duration of the production run. At run time, each system's System Controller coordinates the starting and stopping of runs of material or product by communicating within System to Operation Controllers and also across systems. Some of the aspects of schedule coordination that can be configured are as follows:

1. Modes or criteria for run start such as specified start time, setup complete from prior run, and when any user run has started.

2. Modes or criteria for run end such as end at next start time, specified end time, use the specified goal, first of end time and goal, first of start time for next run and goal, second of end time and goal, all user goals are met, and end when source is empty.

3. Flexibility in determining fate of material trapped in each operation when goal is reached.

4. Coordination of schedules system-to-system, as in the case where an upstream system supplies material to a downstream system.

The coordinating of continuous material production, dictated by a schedule, is a difficult process in the real world. Numerous decisions are made (either by computer or by humans) about how runs of continuous material are to "snake their way" through multiple systems of a production facility.

For example, a foreman receives a schedule (from the Scheduling Department) which tells him that he needs to produce a certain amount of runs of a batch material. A run typically is defined as the same material packaged differently (different label, different size, etc.). He needs 1 run of 100 cases, a run of 200 cases, and another run of 300 cases all from one bin of batch material. He can easily make the first two runs on a packaging line, but on the last run, he has to make the decision sometimes to empty the bin and produce 310 cases rather than 300 .

SDI Industry also allows for the development of custom scheduler modules that can be used to simulate the scheduling department. The scheduler module essentially captures within computer programming, the heuristics (both explicit and vague) which scheduling departments in most manufacturing facilities employ. This type of simulation is done to study scheduling strategies. A user can change some critical inputs to the simulation and quickly generate new test schedules which can be run by the plant simulation. For example, a user could change the target Days Forward Coverage (DFC) for specific brands or change the Demand profile or product mix and quickly test new schedules in the plant simulation.

\section{TYPICAL RESULTS AND REPORTS}

The reporting aspects of SDI Industry are designed to be intuitive and are directly related to production run schedules. This is accomplished via communication with controllers within the hierarchical levels of a model. The modeler can report the results in a customized format with the aid of a Report Generator. Reports generated are incorporated in to the integrated database within SDI Industry.

For each scheduled production run, each system reports production based on the throughput tracking of the Operation Controller in the final operation within a system. This information along with type of product and time of production is used as a basis for generating reports.

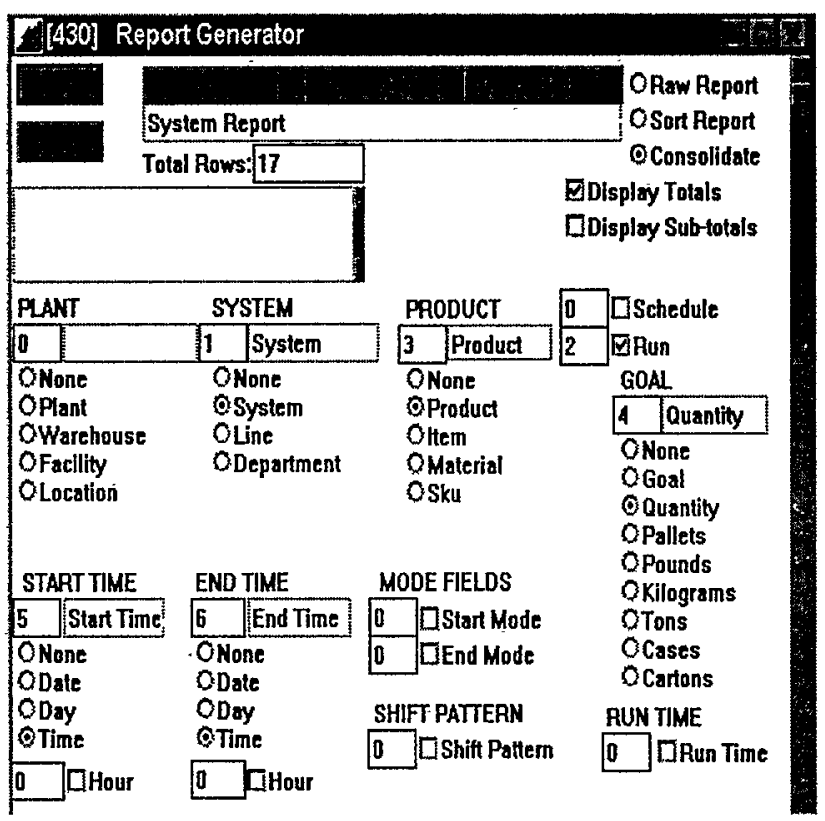

Figure 10: Report Generator

\subsection{Report Generator}

SDI Industry's Report Generator facilitates the organization of this information in the rapid creation of a customized report. Any combination of the fields shown in Figure 10 on the previous page can be used to create a particular report. 


\section{SDI Industry: An Extend-Based Tool for Continuous and High-Speed Manufacturing}

There are various types of reports that can be created with the use of the Report Generator. A raw data report will organize data by production run into a complete listing by order of run completion. This raw data can be sorted by column into numerical order. A consolidated report consolidates rows with the same entry in the first column to single entry with totaled data. An example of a consolidated report is a report by product type. There are generally multiple production runs for one particular product. A consolidated report would tally the data from each run and report it in a single entry for that product.

Each report created in the SDI Industry reporting structure becomes a part of the model database. A System Report created using the Report Generator is shown below in the database display in Figure 11. The columns correspond to fields selected in the creation of the report. In this report, the information is sorted system. The run numbers in the report correspond to the order of the particular production run within the system. With this report, you have a clear display of production results from a model run.

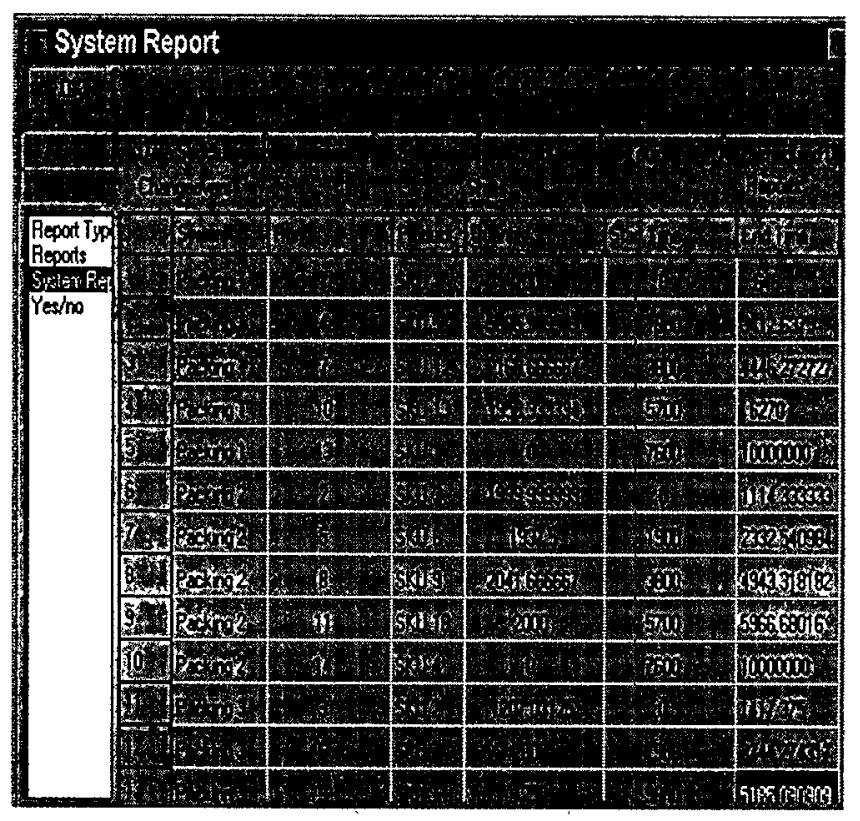

Figure 11: System Report

\subsection{Track Production Interval}

The Track Production Interval (TPI) block provides a tool for reporting production information by time intervals specified by the user. Other dimensions of this report matrix can be product, system, operation or any other category listed in the model database. The model user can insert as many TPI blocks as desired. If two blocks report to the same report, their production amounts will be cumulative.
Specification of reporting intervals may be weekly, daily or by even hour intervals in accordance with predefined formats. There is only one format for weekly reports. Beginning and end of each period is defined by the start time on the model clock. Weeks end exactly seven 24 hour days from the start of the prior week. There are three formats for daily reports - sequential day, day of week and date. Days end exactly 24 hours after the end of the prior day, with the first day starting at the model start time. The day heading is the day on which the period ends. Thus if the model starts at 2am on Monday morning, the first interval will end at 2:00am Tuesday and be called Tuesday, Tuesday's date, or Day 1. The user must enter the number of hours in each interval. There are three format options sequential hour, date and hour, or day and hour. Time periods begin at the time specified in the model clock.

The TPI block will calculate at the end of each period what the production was during that period. The production input connector of this block is connected to a cumulative production output, such as on the top of SDI Industry's Constraint block. The model can control which production category is reported by providing a category number at this connection. This might typically be a product number from an Operation Controller. Note that whatever list of categories is to be used is directly related to the model database.

\section{CONCLUSIONS}

SDI Industry provides a unique new architecture specifically designed to address the needs of bulk and high speed consumer product producers. Simulation Dynamics has been using this tool kit to bring targeted solutions to their clients for over four years. Now SDI Industry brings the power of this architecture to your desktop. A companion product, SDI Supply Chain, is under development for extending this architecture to encompass the entire supply chain of a plant.

\section{REFERENCES}

Krahl, D. 1994. An Introduction to Extend. In Proceedings of the 1994 Winter Simulation Conference, ed. J.D. Tew, S. Manivannan, D.A. Sadowski, and A.F. Seila, 538-545. IEEE, Piscataway, NJ.

Krahl, D. and Lamperti, J. S. 1997, A Message-Based Discrete Event Simulation Architecture. In Proceedings of the 1997 Winter Simulation Conference, ed. S. Andradottir, K. J. Healy, D. H. Withers, and B. L. Nelson, 1361-1367. IEEE, Piscataway, NJ.

Siprelle, A. J. and Phelps, R. A. 1997, Simulation of Bulk Flow and High Speed Operations. In Proceedings of the 1997 Winter Simulation Conference, ed. S. 
Andradottir, K. J. Healy, D. H: Withers, and B. L. Nelson, 706-710. IEEE, Piscataway, NJ.

Siprelle, A. J. and Parsons, D. J. 1995. Modeling a Bulk

Manufacturing System Using Extend. In Proceedings of the 1995 Winter Simulation Conference, ed. J. D. Tew, S. Manivannan, D. A. Sadowski, and A.F: Seila, 813-817. IEEE; Piscataway, NJ.

Sturrock, D. T. and. Drake, G. R. 1996. Simulation for High-Speed Processing. In. Proceedings of the 1996 Winter Simulation; Conference, ed. J. M. Charnes, D. J.s Morrice, D. T. Brunner; and J. J. Swain, 432-436. IEEE, Piscataway, NJ.

\section{AUTHOR BIOGRAPHY}

ANDREW J. SIPRELLE is President and founder of Simulation Dynamics, Inc., a firm that provides simulation consulting, training, and custom models. Mr. Siprelle's industry experience includes creation and analysis of models for strategic and capacity planning, market analysis, and the application of industrial statistics. Applications of simulation have been in the areas: of business, manufacturing, government and service. operations. Before starting Simulàtion Dynamics, Inc. he worked at the. Aluminum Company of America. He received his B.S. in Industrial Engineering and Operations Research from Virginia Polytechnic Institute.

RICHARD A. PHELPS is a Senior Consulting Associate at Simulation Dynamics, Inc.. Mr. Phelps' industrial experience prior to joining SDI includes time in the cutting tool and aluminum industries. He received his B.S. in Industrial and Systems Engineering from the Georgia Institute of Technology where he is a member of the Council of Outstanding Young Alumni.

M. MICHELLE BARNES is a Consulting Associate at Simulation Dynamics. Ms. Barnes industrial experience prior to joining SDI includes work in the automotive industries with Johnson Controls, Allied Signal and TRW. She received her B.S. in Industrial and Manufacturing Engineering from the Tennessee Technological University. 